\title{
Effects of composition on the stability of the magnetic order of ferrimagnetic multilayers
}

\author{
Ana L. Dantas, ${ }^{1,2}$ Selma R. Vieira, ${ }^{3}$ and A. S. Carriço ${ }^{2, *}$ \\ ${ }^{1}$ Departamento de Física, Universidade do Estado do Rio Grande do Norte, Mossoró-RN 59610-210, Brazil \\ ${ }^{2}$ Departamento de Física Teórica e Experimental, Universidade Federal do Rio Grande do Norte, Natal, RN 59072-970, Brazil \\ ${ }^{3}$ Departamento de Física, Universidade Estadual de Feira de Santana, Feira de Santana, BA 44031-460, Brazil
}

(Received 25 January 2002; published 2 May 2002)

\begin{abstract}
We study the field induced instability of the antiferromagnetic phase of magnetic multilayers consisting of a stacking of uniaxial ferromagnetic films, $F_{1}$ and $F_{2}$, with thicknesses $t_{1}$ and $t_{2}$, coupled through a nonmagnetic (NM) spacer. The external field is along the uniaxial axis, in the plane of the films, and the composition is varied by choosing arbitrary values of the relative thickness $\beta=t_{1} / t_{2}$. We calculate the critical value of the external field $H^{*}$, and examine the nature of the phase transition. We find that $H^{*}$ does not change with the multilayer thickness in the case of multilayers with an even number of magnetic layers, $\left(\left[F_{1} / \mathrm{NM} / F_{2} / \mathrm{NM}\right]_{n}\right.$, $n$ arbitrary). The nature of the phase transition and the critical field depend on the value of $\beta$, and the minimum value of $H^{*}$ corresponds to $\beta=1$. For a multilayer with an odd number of magnetic layers we find that the thickness dependence of the critical field is significantly affected by the value of $\beta$.
\end{abstract}

DOI: 10.1103/PhysRevB.65.172414

PACS number(s): 75.70.Ak, 75.70.Cn

Artificial magnetic multilayers composed of ultrathin magnetic films have attracted a considerable research effort in the last decade. Both fundamental aspects and technological applications have motivated research groups worldwide. ${ }^{1}$

The discovery of the giant magnetoresistance ${ }^{2}$ (GMR) motivated a large number of studies of antiferromagnetic (AF) multilayers. ${ }^{3}$ These systems consist in the stacking of thin ferromagnetic $(F)$ films separated by spacers chosen in such a way as to lead to antiferromagnetic interaction between neighboring $F$ films. The GMR results from the modifications in the electrical resistance as the magnetic pattern is changed by an external magnetic field.

One issue which is relevant from the practical point of view is the magnetic stiffness of the multilayer. In this regard a key parameter is the minimum value of the external field strength which produces instability of the intrinsic magnetic order of the multilayer.

The nature of the phase transition is also relevant. In the case of first-order phase transitions the overall change in the magnetic pattern is likely to produce large signatures in the magnetoresistance, since the relative orientation of the magnetic moments of neighboring films may be changed throughout the whole multilayer. A phase transition of second order, with a continuous change in the magnetization, corresponds to small deviations from the intrinsic magnetic order. In this case the change in the electrical resistance is expected to be small.

The possibility of tailoring the GMR curve is of technical interest. The nature of the phase transition affects the field dependence of the GMR. Thus it is worth looking for multilayer parameters that can be controlled macroscopically and yet affect the strength of the threshold magnetic field and the nature of the field induced phase transitions of artificial multilayers.

Previous studies of surface nucleated field induced phase transitions of two sublattice antiferromagnets ${ }^{4-7}$ and transition-metal multilayers ${ }^{8,9}$ addressed the role of the number of layers and the magnetic anisotropy in the stability of the antiferromagnetic phase.
Thin AF films with an odd number $(N)$ of atomic planes require a large external field to make the $\mathrm{AF}$ phase unstable. For large $N$ the surface region is a negligible part of the system and the AF instability occurs at the spin flop field $H_{S F}=\sqrt{2 H_{A} H_{E}+H_{A}^{2}}$, where $H_{E}$ and $H_{A}$ are the exchange and the anisotropy fields. ${ }^{7}$

For AF multilayers made of films with mixed anisotropy the strength of the threshold field can be made rather small and also the nature of the phase transition can be tailored by selecting the relative orientation of the uniaxial anisotropy axis with respect to one of the crystalline anisotropy easy directions. $^{8}$

In most of the examples above ${ }^{4-8}$ the systems consist of the stacking of equivalent layers (or atomic planes), each of which has the same magnetic moment per unit area, the same magnetic anisotropy energy, and also the same effective exchange field. For ferrimagnetic multilayers the stability of the AF phase is controlled by a few more parameters.

The simulation of the magnetic properties of small anisotropy ferrimagnetic multilayers ${ }^{9}$ and reports of experimental studies of ferrimagnetic multilayers ${ }^{10,11}$ revealed new magnetic phases. $\mathrm{Fe} / \mathrm{Gd}$ multilayers have been studied extensively. ${ }^{9}{ }^{12-17}$ In these studies an AF Gd-Fe contact interaction has been used to explain field induced phase transitions as well as thermal effects. A partial Gd twisted state in an epitaxial $\mathrm{Gd} / \mathrm{Fe}$ bilayer was recently reported. ${ }^{18}$ The experimental results were interpreted as resulting from the competing Gd-Gd and a long ranged and oscillatory Gd-Fe exchange interaction. ${ }^{18}$ More recently the spatial extent of the $\mathrm{Gd}-\mathrm{Fe}$ interaction was examined using $\mathrm{Gd}-\mathrm{Fe}$ multilayers. ${ }^{19}$

There are two categories of AF coupled multilayered systems: those with strong direct coupling at the interface and those with weak coupling through nonmagnetic materials. ${ }^{20}$ The antiferromagnetic multilayered systems used in GMR sensors belong in the second category. For these structures the magnetic phases may be described satisfactorily within a self-consistent mean-field approach, ${ }^{21}$ in which each layer is 
represented by an effective spin. A new method to study the equilibrium phases and excitations of multilayers has been proposed recently. ${ }^{22,23}$

In this paper we study ferrimagnetic multilayers in which the intrinsic exchange, within each of the ferromagnetic films, is much larger than the exchange energy coupling adjacent to the films. We study a multilayer composed of ferromagnetic films with two different thicknesses, made of the same material. We show that the magnetic stiffness, as well as the nature of the field induced phase transition, may be tailored by selecting appropriate values of the anisotropy of the ferromagnetic films and the ratio of thicknesses.

The magnetic energy per unit area is given by

$$
E=\sum_{n=1}^{N}\left\{J \vec{S}_{n} \cdot \vec{S}_{n+1}-\frac{K}{2} S_{n z}^{2}-g \mu_{B} S_{n z} H\right\},
$$

where $K$ is the uniaxial anisotropy constant, $g \mu_{B} \vec{S}_{n}$ is the magnetic moment per unit area of the $n$th layer, and $H$ is the strength of the external field applied along the $z$-axis direction. $J$ is the exchange energy coupling neighboring layers. We use $J>0$ and the exchange term is not included for the $N$ th layer. The multilayer composition is changed by taking $S_{n}=\beta S$, if $n$ is odd and $S_{n}=S$ if $n$ is even. We consider 0 $<\beta<1$, so that for $N$ odd the surfaces contain the thinner layer.

The equilibrium structure is found using a method developed earlier and which has been applied in the past for the study of AF films and superlattices, ${ }^{6,7}$ and transition-metal AF multilayers. ${ }^{8,9}$ The method consists in finding the magnetic profile in which the magnetic moment of each layer is along the direction of the local effective field. The local effective field acting in each layer includes the external field, the anisotropy field, and the exchange field. It allows incorporating in a simple manner the lack of coordination at the surfaces as well as the differences in the magnetic moment per unit area between the layers.

We have found that the critical field for multilayers with an even number of layers is not thickness dependent. This is a generalization of previous results ${ }^{8}$ for $\mathrm{AF}$ multilayers $(\beta$ $=1)$. However, the critical field is a function of the multilayer composition. Note from Fig. 1 that the critical field increases as the value of $\beta$ decreases and for $\beta=1$ we find that $H^{*}$ is minimum. For $\beta=1$ the ferrimagnetic multilayer turns into an AF multilayer for which the threshold field is given ${ }^{8}$ by $H_{S S F}=\sqrt{H_{A} H_{E}+H_{A}^{2}}$, where $H_{E}$ and $H_{A}$ are the exchange and anisotropy fields.

A ferrimagnetic multilayer with an even number $N$ of layers has a net magnetic moment per unit area of $(1-\beta)$ $\times(N / 2) M_{S}$, where $M_{S}$ and $\beta M_{S}$ are the magnetic moment per unit area of the layers. For small values of $\beta$ the stabilization due to the Zeeman energy is stronger. When $\beta$ approaches 1 there is no net magnetic moment and the stability of the AF phase decreases. Thus the Zeeman energy contributes to make the multilayers with $\beta<1$ more stable to field effects.

In order to examine how the composition affects the nature of the phase transition, we display in Fig. 2 the total

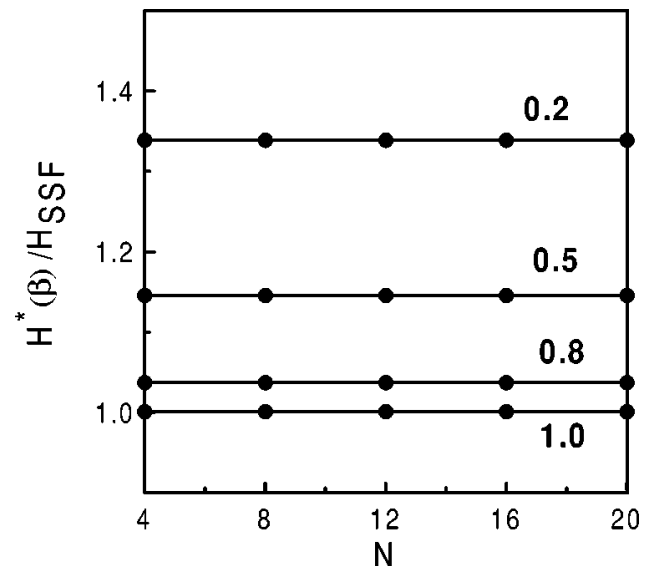

FIG. 1. The critical field for instability of the ferrimagnetic phase of multilayers with even number of layers. The numbers by the curves indicate the values of $\beta, K / 2 J=0.1$ and the critical field is shown in units of the surface spin flop field $H_{S S F}\left[H_{S S F}\right.$ $\left.=H^{*}(\beta=1)\right]$.

magnetic moment of a ten-layer multilayer in the neighborhood of the critical field. We show that for low values of $\beta$ the transition is continuous, while for $\beta \approx 1$ the transition produces a discontinuity in the magnetization. Note from Fig. 1 that the critical field for $\beta=0.2$ is $\approx 30 \%$ larger than that for $\beta=0.8$. In Fig. 2 we use, for each curve, the corresponding critical field, from Fig. 1, as the scale of the field axis.

For $\beta=0.8$ the net magnetization of the multilayer is $M / M_{S}=0.1$, and, within the field interval shown in the picture, there is a variation in the magnetization of around $100 \%$. For $\beta=0.2$ the net magnetization is $M / M_{S}=0.4$ and only a few percent variation in the magnetization is found in the same reduced field interval. Note that in this case the

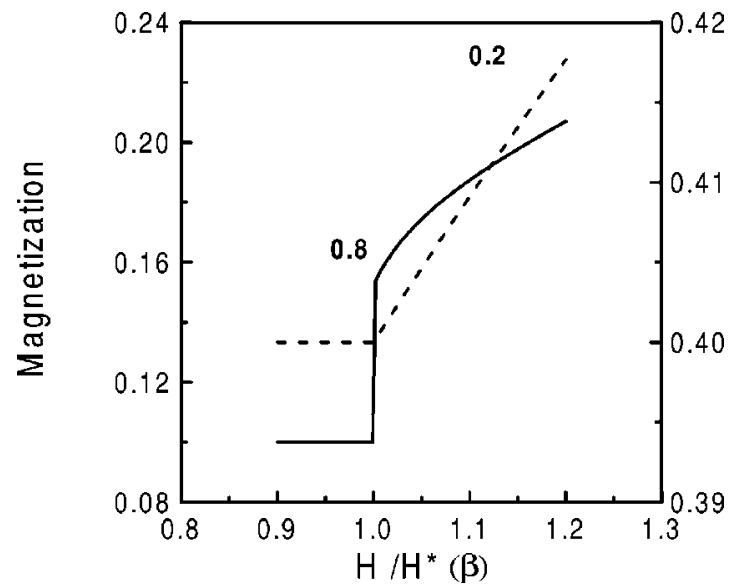

FIG. 2. The total magnetic moment of a ferrimagnetic multilayer with $N=10$. The numbers by the curves indicate the values of $\beta$ and the magnetization is shown in units of the saturation value. The external field is shown in units of the critical field $H^{*}(\beta)$ for the corresponding value of $\beta$. The uniaxial anisotropy is given by $K / 2 J=0.1$. The left-hand side vertical axis is used for the $\beta=0.8$ curve and the right-hand side vertical axis is used for the $\beta=0.2$ curve. 


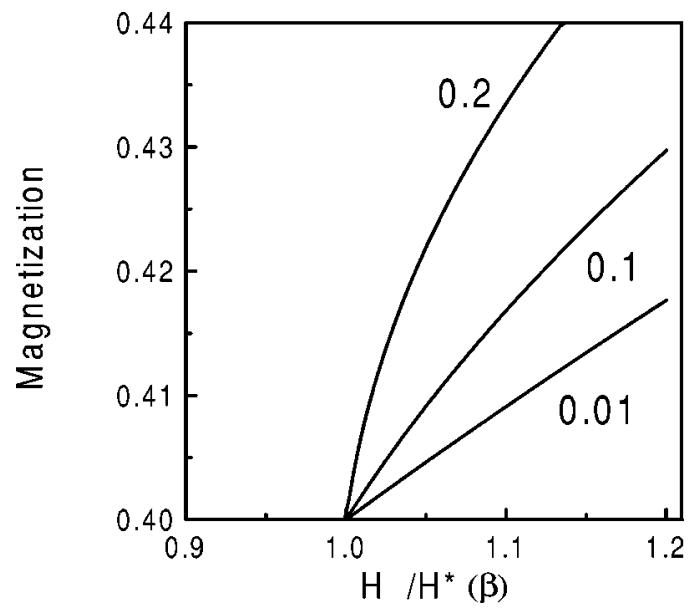

FIG. 3. The total magnetic moment of a ferrimagnetic multilayer with $N=10 . \beta=0.2$ and the numbers by the curves indicate the values of $K / 2 J$. The magnetization is shown in units of the saturation value. The external field is shown in units of the critical field $H^{*}(\beta)$ for $\beta=0.2$ and each value of the anisotropy.

width of the field interval in which the modest variation in magnetization occurs is larger than in the case of $\beta=0.8$, since the critical field for $\beta=0.2$ is larger.

For $\beta=0.2$ the threshold field is large (approximately $\left.1.4 H_{S S F}\right)$. One could expect a spin floplike phase to form. However, due to the large stabilization of the layers with larger magnetic moment, coupled to the external field, the magnetic pattern, at the threshold field, consists of an almost antiferromagnetic arrangement of the layers, with small modifications near the surface with the magnetic moment opposite to the external field. The nearly AF arrangement produces a small magnetization at the transition.

For $\beta=0.8$ the threshold field is close to the surface spin flop field $H_{S S F}$. There is not a large difference between the stability of the layers (the Zeeman energy is almost the same for each of the two sets of layers composing the multilayer) and at the transition a phase similar to a surface spin flop phase is formed. This produces a discontinuity in the magnetization as shown in Fig. 2.

For the chosen value of the anisotropy $(K / 2 J=0.1)$, the modified regions, for both values of $\beta$ involve only a few layers near the surface. For smaller values $K / 2 J$ the modified region extends deeper into the center of the multilayer. We have found that for larger values of $K / 2 J$ (results not shown) one may restrict the modified region to a very small number of layers near the surface of the multilayer. This feature might be of some technological interest, since by controlling the thickness of the region where modifications are made in the original magnetic pattern, might be helpful to tailor the GMR response.

In Fig. 3 we examine the effect of the anisotropy of the layers in the case of a continuous phase transition. We display the magnetization of the multilayer when the external field strength is close to the threshold value. Note from Fig. 3 that the static susceptibility is a function of the anisotropy to exchange ratio $(K / 2 J)$. For small values of $K / 2 J$ the magnetization builds up very slowly at the transition. This might seem contrary to our expectation since the modified surface

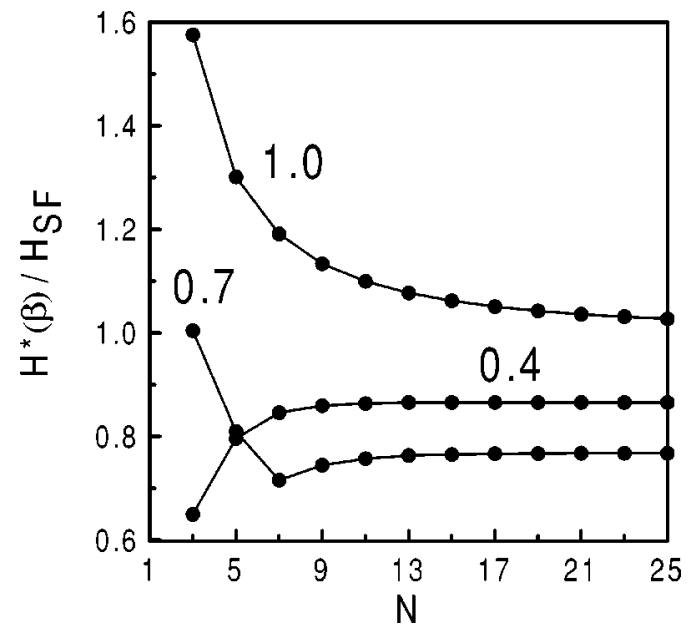

FIG. 4. The critical field for instability of the ferrimagnetic phase of multilayers with an odd number of layers. The numbers by the curves indicate the values of $\beta, K / 2 J=0.1$ and the external field is shown in units of the bulk spin flop field $H_{S F}$.

region increases when the anisotropy decreases. However, for low values of the anisotropy, it costs less energy for the spins to accommodate the Zeeman energy and stay in a nearly AF pattern throughout the multilayer, resulting in a slower increase of the magnetization with the strength of the external field.

In Fig. 4 we show the thickness dependence of the critical field for multilayers with an odd number of layers. We have found that thin multilayers with $\beta<0.5$ have a reduced stability while thin multilayers with $\beta>0.5$ have a critical field of the same order or larger than the spin flop field. We discuss below two typical examples. The curve for $\beta=1.0$, which is that of an AF multilayer ${ }^{7}$ is included for comparison purposes.

For $\beta=0.7$ the critical field of thin multilayers is large and decreases as the multilayer thickness increases, as in the case of AF multilayers. ${ }^{7}$ In this case, for $N<7$, the configuration that minimizes the energy has the surface layers aligned with the external field. Thus the variation in the total magnetic moment per unit area $\left[\delta m=(\beta-1) M_{S}\right]$ between multilayers with $N$ and $N+2$ layers is negative. As the multilayer becomes thicker the Zeeman energy is less efficient in stabilizing the ferrimagnetic order and the threshold field decreases. For multilayers with $N \geqslant 9$ the AF configuration with a lower energy has surface spins opposite to the external field. When $N$ is increased to $N+2$ we find that $\delta m=(1-\beta) M_{S}$ is positive and the threshold field increases.

For $\beta=0.4$ the critical field is an increasing function of the multilayer thickness. In this case thin multilayers become unstable for field strengths smaller than the bulk spin flop field. The equilibrium structure has the surface layers opposite to the external field. The variation of magnetization in going from a $N$-layers multilayer to a $(N+2)$-layers multilayer is positive, and is given by $(1-\beta) M_{S}$. Thus the total magnetization of the multilayer increases when the number of layers is increased. This leads to an extra stability of the ferrimagnetic order as the multilayer becomes thicker and the threshold field increases. 
We have shown that by changing the composition of a ferrimagnetic multilayer made of a single ferromagnetic compound and layers with different thicknesses, one may modify significantly the stability of the magnetic ground state of the system and also the nature of the field induced phase transition. We have also shown that, for odd $N$, the thickness effects on the stability of the ferrimagnetic order have two different regimes, one for $\beta<0.5$ and another for $\beta>0.5$. Some of these features might be of interest for the industrial applications of ferrimagnetic multilayers.

We thank the Brazilian Research Council $\mathrm{CNPq}$ and CAPES for partial financial support.
*Electronic address: acarrico@dfte.ufrn.br

${ }^{1}$ W. J. M. de Jongue, P. J. H. Bloemen and F. J. A. den Boreger, Ultrathin Magnetic Structures, edited by B. Heinrich and A. C. Bland (Springer-Verlag, Berlin, 1994), Vol. I.

${ }^{2}$ M. N. Baibich, J. M. Broto, A. Fert, F. Nguyen Van Dau, P. Pretoff, P. Etiene, G. Creuzet, A. Friedich, and J. Chazelas, Phys. Rev. Lett. 61, 2472 (1988).

${ }^{3}$ J. Nogués and I.K. Schuller, J. Magn. Magn. Mater. 192, 203 (1999); J. M. Daughton, ibid. 192, 334 (1999).

${ }^{4}$ D. L. Mills, Phys. Rev. Lett. 20, 18 (1968).

${ }^{5}$ F. Keffer and H. Show, Phys. Rev. Lett. 31, 1061 (1973).

${ }^{6}$ A. S. Carriço and R. E. Camley, Phys. Rev. B 45, 13117 (1992).

${ }^{7}$ A. S. Carriço, R. E. Camley, and R. L. Stamps, Phys. Rev. B 50, 13453 (1994).

${ }^{8}$ A. L. Dantas and A. S. Carriço, Phys. Rev. B 59, 1223 (1999).

${ }^{9}$ R. E. Camley, Phys. Rev. B 35, 3608 (1987); R. E. Camley and D. R. Tilley, ibid. 37, 3413 (1988).

${ }^{10}$ Y. Kamiguchi, Y. Hayakawa, and H. Fujimori, Appl. Phys. Lett. 55, 1918 (1989).

${ }^{11}$ G. Lauhoff, C. Bruynseraede, J. De Boeck, W. Van Roy, J. A. C. Bland, and G. Borghs, Phys. Rev. Lett. 79, 5290 (1997).

${ }^{12}$ K. Cherifi, C. Dufour, Ph. Bauer, G. Marchal, and Ph. Mangin, Phys. Rev. B 44, 7733 (1991).
${ }^{13}$ M. Sajieddine, Ph. Bauer, K. Cherifi, C. Dufour, G. Marchal, and R. E. Camley, Phys. Rev. B 49, 8815 (1994).

${ }^{14}$ W. Hahn, M. Loewenhaupt, Y. Y. Huang, G. P. Felcher, and S. S. P. Parkin, Phys. Rev. B 52, 16041 (1995).

${ }^{15}$ S. L. Gnatchenko, A. B. Chizhik, D. N. Merenkov, V. V. Eremenko, H. Szymczak, R. Szymczak, K. Fronc, and R. Zuberek, J. Magn. Magn. Mater. 186, 139 (1998).

${ }^{16}$ L. T. Baczewski, R. Kalinowski, and A. Wawro, J. Magn. Magn. Mater. 177, 1305 (1998).

${ }^{17}$ A. Koizumi, M. Takagaki, M. Suzuki, N. Kawamura, and N. Sakai, Phys. Rev. B 61, R14 909 (2000).

${ }^{18}$ O. F. K. McGrath, N. Ryzhanova, C. Lacroix, D. Givord, C. Fermon, C. Miramond, G. Saux, S. Young, and A. Vedyayev, Phys. Rev. B 54, 6088 (1996).

${ }^{19}$ D. Haskel, G. Srajer, J. C. Lang, J. Pollman, C. S. Nelson, J. S. Jiang, and S. D. Bader, Phys. Rev. Lett. 87, 207201 (2001).

${ }^{20}$ B. Dieny and J. P. Gavignan, J. Phys.: Condens. Matter 2, 187 (1990).

${ }^{21}$ R. W. Wang, D. L. Mils, E. E. Fullerton, J. E. Mattson, and S. D. Bader, Phys. Rev. Lett. 72, 920 (1994).

${ }^{22}$ D. L. Mills, J. Magn. Magn. Mater. 198, 334 (1999).

${ }^{23}$ S. Rakhamanova, D. L. Mills, and E. E. Fullerton, Phys. Rev. B 57, 476 (1998). 